\title{
EL CONCEPTO DE SENSIBILIDAD PARA EL CASO DISCRETO DEL PROBLEMA DE REPARTO DE COSTOS DE PRODUCCIÓN*
}

\section{SENSIBILITY CONCEPT FOR THE DISCRETE CASE IN THE COST SHARING PROBLEM}

\author{
Luis HeRnÁNDEZ LAMONEDA ${ }^{\dagger}$ \\ Julio CÉsar Macías PONCE \\ Francisco SÁNChez SÁNChez ${ }^{\S}$
}

Received: 14/Feb/2012; Revised: 16/May/2013; Accepted: 24/May/2013

\footnotetext{
*Este trabajo se realizó con apoyo de CONACyT, proyecto 167924.

${ }^{\dagger}$ CIMAT A.C., Guanajuato, México. E-Mail: lamoneda@cimat.mx

${ }^{\ddagger}$ Universidad Autónoma de Aguascalientes, Aguascalientes, Aguascalientes, México. E-Mail: jlmacias@correo.uaa.mx

${ }^{\S}$ CIMAT A.C., Guanajuato, México. E-Mail: sanfco@cimat.mx
} 


\title{
Resumen
}

Supongamos que se produce un vector en forma conjunta. El problema de reparto de costos es el como dividir el costo de producción de este vector entre sus componentes. En este artículo se caracteriza axiomáticamente una solución para este tipo de problemas basados en dos axiomas: $s$-sensibilidad y separabilidad. El axioma de $s$-sensibilidad establece como debe cambiar la solución cuando ocurren pequeños cambios en la función de costos y el axioma de separabilidad tiene la conotación tradiconal. Se estudia el caso discreto y se obtiene una solución de manera axiomática.

Palabras clave: distribución de costos, soluciones axiomáticas, soluciones inducidas por caminos, juegos cooperativos, sensibilidad.

\begin{abstract}
Suppose that a vector is produced jointly. We will consider the problem of dividing the cost of production among a group of components. In this paper we characterized a solution through two axioms, s-sensibility and separability. The sensibility is a concept related to the variations of a solution due to slight changes of the cost function and the separability is standard. We study the discrete cost sharing problem. We provide a axiomatic solution.
\end{abstract}

Keywords: cost sharing, axiomatic solutions, path-induced solutions, cooperative games, sensibility.

Mathematics Subject Classification: 91A12, 91A40.

\section{Introducción}

En este trabajo estudiamos el problema de reparto de costos de producción en el caso discreto; entendiéndose dicho problema como aquel donde un conjunto de agentes se unen para producir, ya sea porque hacerlo conjuntamente puede abaratar los costos o porque exista la necesidad de producir así. Cada agente tiene una demanda de producción y el tipo de bienes que se generan pueden ser distintos entre ellos. Aunque se puede pensar que se tienen rendimientos crecientes a escala (lo cual motivaría la producción conjunto) no se supone, ni tampoco lo opuesto.

Nosotros solucionamos el problema de manera axiómatica, es decir, pedimos que las soluciones satisfagan un conjunto de supuestos generales que nos permitan rechazar o aceptar soluciones hasta obtener unívocamente la solución. 
Una herramienta apropiada para modelar los problemas de repartición de costos son los llamados juegos de elección multiple, un ejemplo de esto lo tenemos en [3].

La aportación más importante en este artículo es la de relacionar soluciones entre problemas parecidos; así surge el concepto de sensibilidad.

En [1] el lector encontrará soluciones para nuestro problema pero en el caso especial de dos jugadores, ahí se define un juego bipersonal y se obtienen soluciones que son equilibrios de Nash, es trivial verificar que la solución del presente trabajo también es un equilibrio de Nash en aquel contexto.

Por otra parte, en [4] se describen algunos métodos de distribución de costos que se relacionan con nuestro trabajo: los métodos por caminos.

En la siguiente sección, presentamos el modelo matemático y en la Sección 3, mostramos el principal resultado de este trabajo; una caracterización con dos axiomas. En la Sección 4, tenemos las conclusiones.

\section{Preliminares}

\subsection{Definiciones y notación}

En esta sección describimos el modelo matemático de nuestro problema. Sea $N=\{1, \ldots, n\}$ un conjunto finito de agentes, y $m=\left(m_{1}, \ldots, m_{n}\right)$ el vector de demandas, donde cada $m_{i}$ es un entero mayor que cero, que representa la cantidad que requiere producir el agente $i$. Además sea,

$$
M=\left\{0,1, \ldots, m_{1}\right\} \times \cdots \times\left\{0,1, \ldots, m_{n}\right\}
$$

y $M^{o}=M \backslash\{\overline{0}, m\}$ donde $\overline{0}=(0, \ldots, 0) \in \mathbb{R}^{n}$.

Dada la pareja $(N, m)$ la cual supondremos fija a lo largo del trabajo, definimos el espacio de problemas

$$
G_{N, m}=\{c: M \rightarrow \mathbb{R} \mid c(\overline{0})=0\} .
$$

$G_{N, m}$ es el espacio de problemas ${ }^{1}$ para $n$ agentes con vector de demanda $m$. Definiendo la suma y el producto por escalar de manera usual tenemos que $G_{N, m}$ es un espacio vectorial. Una solución es una aplicación

$$
\varphi: G_{N, m} \rightarrow \mathbb{R}^{n}
$$

\footnotetext{
${ }^{1}$ De hecho, a cada función $c$ también se le conoce como función de costos pero durante el desarrollo de este trabajo les llamaremos problemas.
} 
de manera que la componente $\varphi_{i}(c)$ significa el pago asignado al agente $i$. A cada $r=\left(r_{1}, \ldots, r_{n}\right) \in M$ le llamaremos nodo o vector de producción parcial si $r \neq m$ y vector de producción total si $r=m$.

Ejemplo 2.1 Supongamos que en una empresa editorial, se tienen que producir de manera conjunta un tiraje de 3000 unidades de un nuevo libro de texto y 4000 catálogos, el agente 1 es el responsable de la producción de libros mientras que el agente 2 , de los catálogos; las producciones se dan en lotes de millares, es decir $N=\{1,2\}$ y $m=(3,4)$. Los costos de producción por millar en cierta unidad monetaria se listan a continuación

$$
c=\left[\begin{array}{rrrr}
0 & 8 & 14 & 15 \\
12 & 19 & 20 & 25 \\
20 & 22 & 26 & 28 \\
21 & 23 & 30 & 33 \\
24 & 29 & 35 & 40
\end{array}\right]
$$

donde $c(i, j)$ es el costo conjunto de producir $i$ millares de libros para el agente 1 y $j$ millares de catálogos para el agente 2; el costo total de producción conjunta es $c(3,4)=40$. Note que tanto las columnas, como los renglones, se indexan desde cero.

\section{Una solución obtenida con dos axiomas}

El resultado principal de este trabajo es una solución obtenida, pidiendo que esta tenga dos propiedades - $\widehat{s}$-sensibilidad y separabilidad - que a continuación definimos y explicamos.

Primero, presentamos una base para el espacio de problemas. Para cada $r \in M \backslash\{\overline{0}\}$ definimos el problema $E^{r}: M \rightarrow \mathbb{R}$ como sigue:

$$
E^{r}(y):= \begin{cases}1, & \text { si } y=r \\ 0, & \text { si } y \neq r\end{cases}
$$

Es claro que $\left\{E^{r}\right\}_{r \in M \backslash\{\overline{0}\}}$ es una base para el espacio de problemas.

\subsection{Sensibilidad.}

A continuación introducimos el principal concepto en este trabajo.

Sea $s: M^{0} \rightarrow \mathbb{R}^{n}$ una función dada por $s_{k}\left(\left(r_{1}, \ldots, r_{n}\right)\right):=\frac{r_{k}}{m_{k}}$ con $k \in N$. Es decir para cada nodo $r=\left(r_{1}, \ldots, r_{n}\right)$ de producción parcial, $s_{k}$ asigna un valor para el $k$-ésimo agente que es creciente con respecto 
a su producción parcial, por ejemplo si $r_{k}=m_{k}$ entonces el agente $k$ ya cumplió con su demanda total de producción por lo que $r$ sería un nodo muy importante para él, y así $s_{k}$ asignaría el valor de 1 .A su vez si existe $j$ tal que $r_{j}=0$ entonces el agente $j$ podría tener poco interés por el nodo $r$, en este caso el valor de $s_{k}$ sería de cero.

Se pueden definir más funciones como la anterior que modelen criterios de "asignación de importancia" a los nodos por parte de los agentes, por ejemplo,si se consideran ahora la proporción de unidades producidos en el nodo $r$ con respecto a la demanda total $\sum_{i \in N} m_{i}$ o a la producción parcial conjunta $\sum_{i \in N} r_{i}$ tendríamos respectivamente las funciones $s^{1}$ y $s^{2}$ definidas por

$$
s_{i}^{1}(r)=\frac{r_{i}}{m_{1}+\ldots+m_{n}} \quad y \quad s_{i}^{2}(r)=\frac{r_{i}}{r_{1}+\ldots+r_{n}} .
$$

Al conjunto de todas las funciones con dominio $M^{0}$ y contradominio $\mathbb{R}^{n}$ lo denotaremos con $S$ y a cada $s \in S$ le llamaremos una función de sensibilidad y $s_{k}(r)$ se interpreta como la valoración que el $k$-ésimo agente le está dando al nodo $r$.

Ahora estamos en condiciones de definir soluciones $s$-sensibles:

Definición 3.1 Sea $\varphi: G_{N, m} \rightarrow \mathbb{R}^{n}$ una solución. Dada $s \in S$, decimos que $\varphi$ es s-sensible, si para todo $c \in G_{N, m}$,

$$
\frac{\partial \varphi(c)}{\partial E^{r}}=s(r) \forall r \in M^{o} \text { donde } \frac{\partial \varphi(c)}{\partial E^{r}}:=\lim _{h \rightarrow 0} \frac{\varphi\left(c+h E^{r}\right)-\varphi(c)}{h} .
$$

Esta propiedad indica que la variación de $\varphi$ en cada nodo distinto de $m$ queda determinado por $s$. En particular, si $\varphi$ es una solución lineal, entonces $\varphi\left(E^{r}\right)=s(r) \forall r \in M^{o}$.

\subsubsection{Soluciones $s$-sensibles}

Dadas $s \in S$ y $c \in G_{N, m}$, definimos:

$$
\varphi^{h, s}(c):=h(c(m))+\sum_{r \in M^{o}} c(r) s(r)
$$

donde $h$ es una función arbitraria.

Teorema 3.1 Dada $s \in S, \varphi$ es s-sensible sí y sólo sí $\varphi=\varphi^{h, s}$, para alguna $h: \mathbb{R} \rightarrow \mathbb{R}^{n}$. 
Prueba: Si $\varphi(c)=h(c(m))+\sum_{x \in M^{0}} c(x) s(x)$ para alguna $h$, entonces, si $r \in M^{0}$

$$
\frac{\partial \varphi}{\partial E^{r}}(c)=s(r)
$$

Para el recíproco, supongamos que $\varphi=\left(\varphi_{1}, \ldots, \varphi_{n}\right)$ es $s$-sensible.

En primer lugar, observe que si $f: \mathbb{R}^{k} \rightarrow \mathbb{R}$ es una función diferenciable, tal que para toda $x=\left(x_{1}, \ldots, x_{k}\right) \in \mathbb{R}^{k}$ se tiene que $\frac{\partial f}{\partial x_{r}}(x)=s_{r}$, $r: 1, \ldots, k-1$, donde las $s_{r}$ son constantes, entonces -por el teorema del valor medio de cálculo-

$$
f\left(x_{1}, \ldots, x_{k}\right)=s_{1} x_{1}+\cdots+s_{k-1} x_{k-1}+g\left(x_{k}\right)
$$

para alguna función diferenciable $g$. Ahora, $\frac{\partial \varphi}{\partial E^{r}}(c)=s(r)$, para toda $c$ y para toda $r \in M^{0}$, se traduce en que para cada $i: 1, \ldots, n$

$$
\frac{\partial \varphi_{i}}{\partial E^{r}}(c)=s_{i}(r)
$$

y por lo tanto, según lo anterior

$$
\varphi_{i}(c)=\sum_{r \in M^{0}} c(r) s_{i}(r)+h_{i}(c(m))
$$

para alguna función diferenciable $h_{i}$.

Corolario $3.1 \varphi$ es lineal y s-sensible si, y sólo si $\varphi=\varphi^{h, s}$ con h lineal.

\subsection{Separabilidad}

Existen problemas en los que los agentes no se benefician, ni se perjudican, con la producción conjunta, es decir, el costo de la producción conjunta sólo es la suma de los costos individuales cuando producen por separado. Si este es el caso, pediremos que la solución para el caso de la producción conjunta, sea que cada quien pague lo suyo.

Definición 3.2 Se dirá que $c \in G_{N, m}$ es un problema con costos independientes entre agentes si existen funciones $f_{1}, \ldots, f_{n}: \mathbb{R} \rightarrow \mathbb{R}$ con $f_{i}(0)=0$, tales que

$$
c(r)=\sum_{i \in N} f_{i}\left(r_{i}\right)
$$

para todo $r=\left(r_{1}, \ldots, r_{n}\right) \in M$. 
Definición 3.3 Se dirá que $\varphi$ es separable si para todo problema con costos independientes c (como en la definición anterior), se cumple que

$$
\varphi(c)=\left(f_{1}\left(m_{1}\right), \ldots, f_{n}\left(m_{n}\right)\right) .
$$

Si los costos son independientes entre los agentes, entonces cada agente paga el costo de su producción.

\subsection{Sensibilidad inducida por caminos}

Sea $m=\left(m_{1}, \ldots, m_{n}\right)$, como antes, el vector de demandas y denotemos por $m(N)=m_{1}+\ldots+m_{n}$. Supongamos que se tiene una secuencia de producción la cual consiste de $m(N)$ etapas. En cada etapa se produce uno de los artículos de alguno de los agentes. Un pago "natural" asociado a dicha secuencia, sería que cada agente pagara la suma de los costos marginales de sus productos. A continuación explicamos con más detalle lo que significa.

Definimos primero el concepto de camino: un camino es una función

$$
\pi:\{0,1, \ldots, m(N)\} \rightarrow M
$$

con $\pi(0)=\overline{0}, \pi(m(N))=m$ y tal que para todo $t \in\{1,2, \ldots, m(N)\}$ se cumpla que: $\pi(t)-\pi(t-1)=e^{i}$ para algún $i \in N$ donde $e^{i}$ es el $i$-ésimo vector de la base canónica en $\mathbb{R}^{n}$. Debemos ver a un camino como una secuencia de producción; en el Ejemplo 2.1 un camino es el siguiente (ver Figura 1):

$$
\begin{aligned}
& \pi(0)=(0,0) \quad \pi(1)=(0,1) \quad \pi(2)=(1,1) \quad \pi(3)=(2,1) \\
& \pi(4)=(3,1) \quad \pi(5)=(3,2) \quad \pi(6)=(3,3) \quad \pi(7)=(3,4)
\end{aligned}
$$

lo que significa que el agente 2 hace el primer producto, luego el agente 1 hace consecutivamente tres productos y finalmente, el agente 2 hace tres productos más, para completar el total.

Al conjunto de caminos lo denotamos con $\Pi$. Nótese que la cardinalidad de $\Pi$ es $|\Pi|=\frac{m(N) !}{m_{1} ! \cdots m_{n} !}$.

Dado un camino $\pi$, tenemos que el artículo $t$-ésimo en ese camino es producido por el agente $k$ si $\pi(t)-\pi(t-1)=e^{k}$ luego entonces decimos 


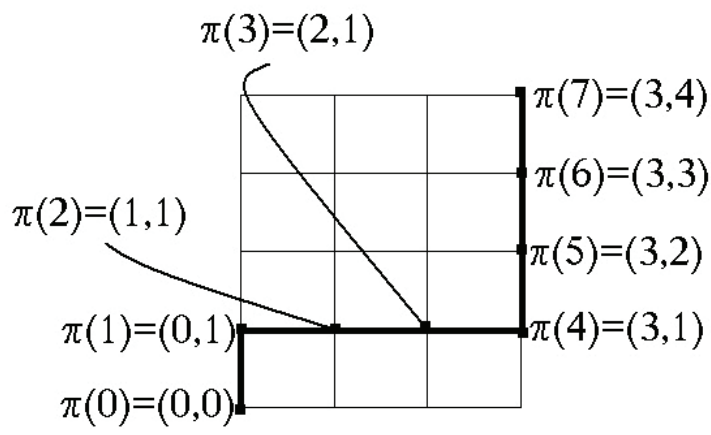

Figura 1: Ejemplo de secuencia de producción

que el costo marginal del $t$ - ésimo producto es $c(\pi(t))-c(\pi(t-1))$, si asignamos este costo al agente $k$ y luego sumamos todos los costos marginales por agente estaremos definiendo una solución $\varphi^{\pi}: G_{N, m} \rightarrow \mathbb{R}^{n}$ dada por:

$$
\varphi^{\pi}(c)=\sum_{t=1}^{m(N)}[c(\pi(t))-c(\pi(t-1))][\pi(t)-\pi(t-1)] ;
$$

Observe que $\varphi^{\pi}$ es lineal. En el caso del camino $\pi$ de la figura 1 y para el problema $c$ del Ejemplo 2.1, se tiene que $\varphi^{\pi}(c)=[12-0](0,1)+$ $[19-12](1,0)+[20-19](1,0)+[25-20](1,0)+[28-25](0,1)+$ $[33-28](0,1)+[40-33](0,1)=(13,27)$.

Sea $r$ un nodo y $\pi$ un camino que pasa por $r$ al tiempo $t_{0}$, esto es, $\pi\left(t_{0}\right)=r$; entonces, si $0<t_{0}<m(N)$ tenemos la siguiente situación (ver Figuras 2 y 3 ):

$$
\pi\left(t_{0}\right)-\pi\left(t_{0}-1\right)=\pi\left(t_{0}+1\right)-\pi\left(t_{0}\right)
$$

O

$$
\pi\left(t_{0}\right)-\pi\left(t_{0}-1\right) \neq \pi\left(t_{0}+1\right)-\pi\left(t_{0}\right) .
$$

En el primer caso tenemos que un mismo agente esta produciendo dos artículos de manera consecutiva mientras que en el segundo hay dos agentes distintos (digamos $l$ y $j$ ).

En particular, para el problema $E^{r}$, tenemos que $\varphi^{\pi}\left(E^{r}\right)=0$ en el primer caso mientras que para el segundo, $\varphi^{\pi}\left(E^{r}\right)=e^{l}-e^{j}$ donde hemos supuesto que $\pi\left(t_{0}\right)-\pi(t-1)=e^{l}$ y $\pi\left(t_{0}+1\right)-\pi\left(t_{0}\right)=e^{j}$. Luego, en este 

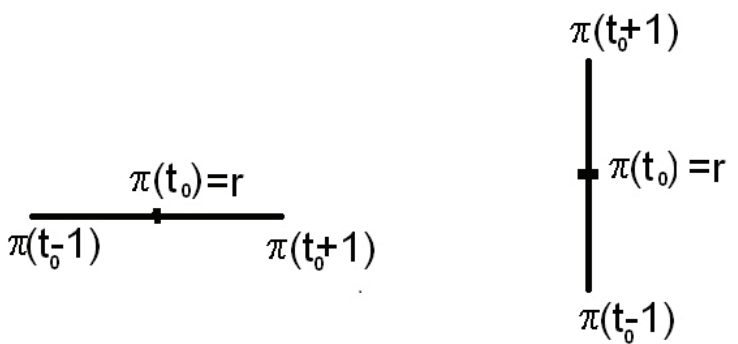

Figura 2: Primera situación posible
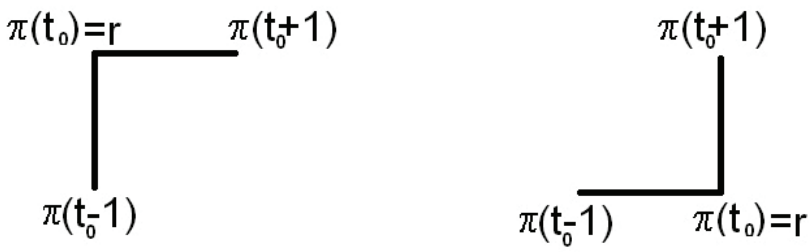

Figura 3: Segunda situación posible

ultimo caso, el agente $l$ paga 1 mientras que el agente $j$ recibe 1 (paga -1). Así para cada $k \in N$ y $r \in M^{o}$ definimos los conjuntos:

$$
D_{k}(r)=\left\{\pi \in \Pi \mid \varphi_{k}^{\pi}\left(E^{r}\right)=1\right\}
$$

y

$$
A_{k}(r)=\left\{\pi \in \Pi \mid \varphi_{k}^{\pi}\left(E^{r}\right)=-1\right\} .
$$

Obsérvese que $\pi \in D_{k}(r)$ significa que el agente $k$ paga 1 en el problema $E^{r}$ usando el camino $\pi$ mientras que $\pi \in A_{k}(r)$ implicaría que el mismo agente recibiría 1 para el mismo problema.

Ahora, definimos la función $\widehat{s}$ como sigue: $\widehat{s}: M^{o} \rightarrow \mathbb{R}^{n}$, tal que:

$$
\widehat{s_{k}}(r)=\frac{1}{|\Pi|}\left[\left|D_{k}(r)\right|-\left|A_{k}(r)\right|\right]
$$

a $\widehat{s}$ le llamaremos sensibilidad inducida por caminos.

$\widehat{s_{k}}(r)$ esta contando para el problema $E^{r}$ la proporción de la diferencia (para el agente $k$ ) de caminos desfavorables (pago de +1 ) vs caminos favorables (pago de -1 ) con respecto al total de caminos. Así $\widehat{s}$ esta valorando (para todos los caminos) que implicaciones tiene pasar por cada uno de los nodos (el nodo $r$ se analiza en el problema básico $E^{r}$ ); dependiendo del 
nodo, cada agente identifica caminos buenos, malos e indiferentes. A continuación veremos como esta función de sensibilidad $\widehat{s}$ induce una solución que promedia a lo largo de todos los caminos.

\subsection{Una solución}

El principal resultado de este artículo es un valor caracterizado con dos axiomas: $\widehat{s}$-sensibilidad y separabilidad. El teorema más importante del trabajo es el siguiente:

Teorema 3.2 Sea $\widehat{s}$ la función de sensibilidad definida anteriormente entonces existe una única solución que satisface $\widehat{s}$-sensibilidad y separabilidad. Dicha solución esta dada por:

$$
\psi(c):=\frac{c(m)}{m(N)} m+\sum_{r \in M^{o}} c(r) \widehat{s}(r) .
$$

De hecho, $\psi$ coincide con la solución que se obtiene de promediar los costos marginales inducidos por los caminos. A saber,

$$
\psi(c)=\frac{1}{|\Pi|} \sum_{\pi \in \Pi} \varphi^{\pi}(c) .
$$

Antes de ver la demostración, notemos que el término $\frac{c(m)}{m(N)} m$ se puede interpretar como un reparto de costos proporcional a la demanda y la suma $\sum_{r \in M^{o}} c(r) \widehat{s}(r)$ como el término de "corrección" 2 que depende de $\widehat{s}$ y de los nodos.

Prueba: Mostraremos que

$$
\psi(c):=\frac{c(m)}{m(N)} m+\sum_{r \in M^{o}} c(r) \widehat{s}(r)
$$

es $\widehat{s}$-sensible y separable, que es única y que

$$
\frac{c(m)}{m(N)} m+\sum_{r \in M^{o}} c(r) \widehat{s}(r)=\frac{1}{|\Pi|} \sum_{\pi \in \Pi} \varphi^{\pi}(c) .
$$

Primero mostramos la última aserción; Para cualquier $\pi \in \Pi$ y $x \in M^{o}$, ya vimos que $\varphi^{\pi}\left(E^{x}\right)=0$ o $\varphi^{\pi}\left(E^{x}\right)=e^{l}-e^{j}$ para algún $l$ y $j$ en $N$ con

\footnotetext{
${ }^{2}$ Se esta compensando (y acumulando con la suma) a cada jugador, en cada nodo, de manera proporcional al costo de producción parcial, el factor de proporcionalidad es precisamente la importancia del nodo (definida por la función de sensibilidad $\widehat{s}$ )
} 
$l \neq j$. Así, $\sum_{\pi \in \Pi} \varphi_{i}^{\pi}\left(E^{x}\right)=\left|D_{i}(x)\right|-\left|A_{i}(x)\right|$, para todo $i \in N$. Luego entonces

$$
\begin{aligned}
\psi_{i}\left(E^{x}\right) & =\frac{E^{x}(m)}{m(N)} m_{i}+\sum_{r \in M^{o}} E^{x}(r) \widehat{s_{i}}(r)=\widehat{s_{i}}(x)= \\
& =\frac{\left|D_{i}(x)\right|-\left|A_{i}(x)\right|}{|\Pi|}=\frac{1}{|\Pi|} \sum_{\pi \in \Pi} \varphi_{i}^{\pi}\left(E^{x}\right)
\end{aligned}
$$

Si $x=m$ entonces

$$
\psi_{i}\left(E^{m}\right)=\frac{E^{m}(m)}{m(N)} m_{i}+\sum_{r \in M^{o}} E^{m}(r) \widehat{s}_{i}(r)=\frac{1}{m(N)} m_{i}=\frac{1}{|\Pi|} \sum_{\pi \in \Pi} \varphi_{i}^{\pi}\left(E^{m}\right) .
$$

La ultima desigualdad se sigue del hecho de que $\varphi_{i}^{\pi}\left(E^{m}\right)=1$ si $\pi$ es un camino que pasa por el nodo $m-e^{i}$ (para el resto de los caminos el valor es cero) y en total hay $\frac{[m(N)-1] !}{m_{1} ! \cdots\left(m_{i}-1\right) ! \cdots m_{n} !}$ de estos caminos, si multiplicamos por $\frac{1}{|\Pi|}$ obtenemos $\frac{1}{m(N)} m_{i}$. iguales.

Como $\psi$ y $\frac{1}{|\Pi|} \sum_{\pi \in \Pi} \varphi_{i}^{\pi}$ son ambas lineales y coinciden en la base, son

A continuación vamos a probar que $\psi$ satisface separabilidad. Para ello usaremos la igualdad $\psi(c)=\frac{1}{|\Pi|} \sum_{\pi \in \Pi} \varphi^{\pi}(c)$ que se probo anteriormente.

Sea, entonces, $c$ un problema con costos independientes entre agentes, dado por $c(y)=\sum_{i \in N} f_{i}\left(y_{i}\right)$, entonces, para cada $\pi \in \Pi$,

$$
\begin{aligned}
\varphi^{\pi}(c) & =\sum_{t=1}^{m(N)}[c(\pi(t))-c(\pi(t-1))][\pi(t)-\pi(t-1)]= \\
& =\sum_{t=1}^{m(N)}\left[\sum_{i \in N}\left[f_{i}\left(\pi_{i}(t)\right)-f_{i}\left(\pi_{i}(t-1)\right)\right]\right][\pi(t)-\pi(t-1)] .
\end{aligned}
$$

Para cada $k \in N$, sea $T_{k}=\left\{t \mid \pi(t)-\pi(t-1)=e^{k}\right\}$ entonces $\varphi_{k}^{\pi}(c)=\sum_{t \in T_{k}}\left[\sum_{i \in N}\left[f_{i}\left(\pi_{i}(t)\right)-f_{i}\left(\pi_{i}(t-1)\right)\right]\right]$ obsérvese que $i \neq k$ implica que

$f_{i}\left(\pi_{i}(t)\right)=f_{i}\left(\pi_{i}(t-1)\right)$, luego:

$$
\varphi_{k}^{\pi}(c)=\sum_{t \in T_{k}}\left[f_{k}\left(\pi_{k}(t)\right)-f_{k}\left(\pi_{k}(t-1)\right)\right]=f_{k}\left(m_{k}\right)-f_{k}(0)=f_{k}\left(m_{k}\right)
$$


así que

$$
\varphi^{\pi}(c)=\left(f_{1}\left(m_{1}\right), \ldots, f_{n}\left(m_{n}\right)\right)
$$

y como esta igualdad es válida para todo $\pi \in \Pi$, entonces:

$$
\psi(c)=\frac{1}{|\Pi|} \sum_{\pi \in \Pi} \varphi^{\pi}(c)=\left(f_{1}\left(m_{1}\right), \ldots, f_{n}\left(m_{n}\right)\right) .
$$

Observe que el Teorema 3.1 implica que $\psi$ es $\widehat{s}$-sensible. La unicidad se sigue también del Teorema 3.1: Sea $\varphi^{h, \widehat{s}}$ una solución $\widehat{s}$-sensible y supongamos que también es separable. Sea $t \in \mathbb{R}$ arbitrario, entonces para el problema de costos independientes $c$, con

$$
f_{1}(k)=\left\{\begin{array}{cc}
0, & \text { si } k<m_{1} \\
t, & \text { si } k=m_{1}
\end{array}\right.
$$

y $f_{2}=\ldots=f_{n}=0$.entonces por separabilidad

$$
(t, 0, \ldots, 0)=\varphi^{h, \widehat{s}}(c)=h(t)+\sum_{r \neq m \mid r_{1}=m_{1}} t \widehat{s}(r)
$$

lo cual implica que a lo más existe una forma de elegir la función h.

Observe que si definimos las soluciones $\theta$ y $\omega$ por

$$
\theta(c)=\sum_{r \in M^{o}} c(r) \widehat{s}(r) \text { y } \quad \omega(c)=\left(\omega_{1}(c), \ldots, \omega_{n}(c)\right)
$$

donde $\omega_{i}(c)=c\left(0, \ldots, m_{i}, \ldots 0\right)$; trivialmente se sigue que $\theta$ es una solución $\widehat{s}$-sensible pero no separable mientras que $\omega$ es separable pero no $\widehat{s}$-sensible. Así que hay independencia entre los axiomas.

\section{Conclusiones}

Comenzando con la noción de sensibilidad y separabilidad, propusimos una función de sensibilidad $\widehat{s}$ y obtuvimos la solución de los promedios de los caminos, que denotamos con $\psi$, la cual corrresponde a la solución del Método de Aumman-Shapley ([2]) para el problema general discreto de reparto de costos y también corresponde a la solución de Nouweland en el contexto de juegos de elección multiple.

Es importante señalar que en este trabajo no estamos suponiendo que la función de costos sea creciente. Otro resultado útil que obtuvimos es la expresión de las soluciones $s$-sensibles para cualquier función de sensibilidad $s$. En particular, debemos observar que a lo más existe una solución 
$s$-sensible y separable para cada $s$. Quizás, en algunos casos, dada $s$, no exista una solución $s$-sensible y separable.

La función de sensibilidad $\widehat{s}$ nos indujo la solución de los promedios, lo que puede significar que tenemos una adecuada función de sensibilidad, sin embargo, un nuevo problema de investigación es buscar más funciones de sensibilidad, incluso dar una axiomatización en segundo grado que determine una única función de sensibilidad. Además a partir de este trabajo podemos identificar las funciones de sensibilidad para las soluciones existentes en la literatura, por ejemplo, las soluciones igualitaria $\left(\varepsilon_{i}(c):=\frac{c(m)}{N}\right)$ y proporcional $\left(\eta_{i}(c):=\frac{c(m) m_{i}}{m_{1}+\ldots m_{n}}\right)$ son cero-sensibles es $\operatorname{decir} s \equiv 0$.

\section{Referencias}

[1] Macías, J.; Olvera, W. (2012) "A Nash equilibrium solution for the discrete two-person cost sharing problem", Applied Mathematical Sciences 6(42): 2063-2070.

[2] Moulin, H. (1995) "On additive methods to share joint costs", The Japanese Economic Review 46: 303-332.

[3] Nouweland, A.; Tijs, S. (1995) "Cores and related solution concepts for multi-choice games", Mathematical Methods of Operations Research 41: 289-311.

[4] Sprumont, Y. (2008) "Nearly serial sharing methods", International Journal of Game Theory 37: 155-184. 
